\title{
Learning speech recognition from songbirds
}

\author{
Izzet B Yildiz ${ }^{1 *}$, Katharina von Kriegstein ${ }^{1}$, Stefan J Kiebel ${ }^{1,2}$ \\ From Twenty Second Annual Computational Neuroscience Meeting: CNS*2013 \\ Paris, France. 13-18 July 2013
}

Our knowledge about the computational mechanisms underlying human learning and recognition of speech is still very limited [1]. One difficulty in deciphering the exact means by which humans recognize speech is that there are scarce experimental findings at a neuronal, microscopic level. Here, we show that our neuronalcomputational understanding of speech learning and recognition may be vastly improved by looking at a different species, i.e., the songbird, which faces the same challenge as humans: to learn and decode complex auditory input partitioned into sequences of syllables, in an online fashion [2]. Motivated by striking similarities between the human and songbird neural recognition systems at the macroscopic level $[3,4]$, we assumed that the human brain uses the same computational principles at a microscopic level and translated a birdsong model [5] into a human speech learning and recognition model. The model performs a Bayesian version of dynamical, predictive coding [6] based on an internal generative model of how speech dynamics are produced. This generative model consists of a two-level hierarchy of recurrent neural networks similar to the song production hierarchy of songbirds [7]. In this predictive coding scheme, predictions about the future trajectory of the speech stimulus are dynamically formed based on a learned repertoire and the ongoing stimulus. The hierarchical inference uses top-down and bottom-up messages, which aim to minimize an error signal, the so-called prediction error.

We show that the resulting neurobiologically plausible model can learn words rapidly and recognize them robustly, even in adverse conditions. Also, the model is capable of dealing with variations in speech rate and competition by multiple speakers. In addition, we show that recognition can be performed even when words are spoken by different speakers and with different accents-an everyday situation in which current state-of-the-art speech recognition models often fail. We use the model to provide computational explanations for inter-individual differences in accent adaptation, as well as age of acquisition effects in second language learning. For the latter, we qualitatively modeled behavioral results from an experimental study [8].

\section{Author details}

${ }^{1}$ Max Planck Institute for Human Cognitive and Brain Sciences, Leipzig, 04103 Germany. ${ }^{2}$ Biomagnetic Center, Hans Berger Clinic for Neurology, University Hospital Jena, Friedrich-Schiller-University Jena, 07747, Germany.

Published: 8 July 2013

\section{References}

1. Hickok G, Poeppel D: Opinion - The cortical organization of speech processing. Nat Rev Neurosci 2007, 8(5):393-402.

2. Prather JF, Nowicki S, Anderson RC, Peters S, Mooney R: Neural correlates of categorical perception in learned vocal communication. Nat Neurosci 2009, 12(2):221-228.

3. Bolhuis JJ, Okanoya K, Scharff C: Twitter evolution: converging mechanisms in birdsong and human speech. Nat Rev Neurosci 2010, 11(11):747-759.

4. Doupe AJ, Kuhl PK: Birdsong and human speech: Common themes and mechanisms. Annu Rev Neurosci 1999, 22:567-631.

5. Yildiz IB, Kiebel SJ: A Hierarchical Neuronal Model for Generation and Online Recognition of Birdsongs. Plos Comput Biol 2011, 7(12):e1002303.

6. Friston KJ, Trujillo-Barreto N, Daunizeau J: DEM: A variational treatment of dynamic systems. Neuroimage 2008, 41(3):849-885.

7. Fee MS, Kozhevnikov AA, Hahnloser RHR: Neural mechanisms of vocal sequence generation in the songbird. Annals of the New York Academy of Sciences 2004, 1016:153-170.

8. Meador D, Flege JE, Mackay IRA: Factors affecting the recognition of words in a second language. Bilingualism: Language and Cognition 2000, 3:55-67.

doi:10.1186/1471-2202-14-S1-P210

Cite this article as: Yildiz et al:: Learning speech recognition from songbirds. BMC Neuroscience 2013 14(Suppl 1):P210.

\footnotetext{
* Correspondence: yildiz@cbs.mpg.de

${ }^{1}$ Max Planck Institute for Human Cognitive and Brain Sciences, Leipzig,

04103 Germany

Full list of author information is available at the end of the article
}

(c) 2013 Yildiz et al; licensee BioMed Central Ltd. This is an Open Access article distributed under the terms of the Creative Commons Attribution License (http://creativecommons.org/licenses/by/2.0), which permits unrestricted use, distribution, and reproduction in any medium, provided the original work is properly cited. 